\title{
Summary Design on Curriculum Management System
}

\author{
Li Ma \\ School of Education and Sports, Bohai University, Jinzhou, 121013, China \\ 88228471@qq.com
}

Keywords: curriculum management system; summary design; design strategy; architecture design; functional design

\begin{abstract}
Curriculum management is an important educational administration work, involving curriculum arrangement and performance management and so on, is one of the most complex work of educational administration. This article follows the principle and method of system engineering for summary design and lay the foundation for system development. Design process based on the summary design strategy, mainly completed the system architecture design and system functional design. Architecture design adopts three layer structure, the presentation layer directly interact with the user, the business layer is used to handle user input information, data layer definition and maintain data integrity and security; Functional design using modular method, designed by the Web page, course management, system utilities, rights management, basic information and query statements such as module and its logical structure of sub modules.
\end{abstract}

\section{Introduction}

Office is the most important and the most onerous position, is also the most can reflect the characteristics of school teaching departments, colleges and universities teaching affairs management level directly reflects the overall teaching level. Traditional way of artificial management there are a lot of repeated labor, low productivity, information update disadvantages such as not in time, can't meet the need of the development of the information age, unable to meet the needs of modern teaching management. Therefore, around the content and goals of teaching affairs, on the basis of learning teaching plan, integration of educational administration process, reasonable use of existing resources, develop a modern educational administration information management system, can effectively solve the plight of educational administration work, improve the level of teaching management. Development of university educational administration management system, to realize information sharing within the scope of the whole school teaching, the teachers and students through the network for teaching information and resources, for the majority of teachers and students to provide quality and efficient teaching environment and good service guarantee. At the same time, the existing hardware and software resources and data to effectively integrate resources, change the traditional way of office, for building interconnectivity, efficient Shared, unified management of the digital platform foundation.

Summary design is designed according to user interaction process and user needs to create the interaction framework and visual framework of process, the result is often to reflect the interaction controls the overall layout, interface elements grouping and interface board type to present in the form of a page frame. Summary design and requirements analysis is the process of the production function model, data model and behavior model analysis conclusion, the conclusions of the resulting design process. In the transformation process from analysis to design, summary design to produce the relevant software system architecture, software architecture and data structure design model. These conclusions will be written into the general design document, the late as the basic foundation of detailed design, and to provide technical solution for the detailed design and program code. Curriculum management is an important educational administration work, involving curriculum and performance management aspects of problem, is one of the most complex work in the educational administration [1,2]. Curriculum management system is an important component of the comprehensive educational administration system, this paper carries on the summary design, foundation for later the detailed design and software implementation. 


\section{Summary Design Strategy}

Structured design is a method of top-down, decompose step by step, through the module chart to represent the logical structure of the software. Module is a certain function, can be individually named accessed data and a collection of program statements. In combination with the ideas and methods of software engineering, using structured approaches for software summary design should follow the following strategies:

(1) Module has a relatively independent [3]. Module independence refers to each module in the software system has the function of single and don't have much contact with other module. Each module in the software system, namely, only the software requirements of a particular function, and other modules in the software system interface is simple, to reduce unnecessary data cross, calls and control.

(2) The module partition should facilitate system implemented in stages. Every stage of life cycle of the software development process based on the activities of the division, on the basis of user requirements can be converted to the software system the process of collection activities. According to the theory of the life cycle of software engineering, software development is a complicated system engineering, the development process is typically in stages, module partition should consider the request.

(3) Reduce the module interface complexity and redundancy. Complex is a software module interface error, a main reason of the module interface should be as simple as possible and is consistent with the module function. Interface complex or inconsistent (i.e., there has been no contact between transfer of data) and characterization of high coupling and low cohesion, to analyze the independence of the module.

(4) Modules can be predicted. Module function constant predictable refers to input, output constant, should also be based on the input data to predict the output. In addition, if the design of the local data structure of the module, the control process and other factors, such as external interface limit too much, to remove these restrictions will increase maintenance costs later.

\section{System Architecture Design}

With the continuous progress of software engineering and specifications, and the application of object-oriented programming ideas, people, extension of encapsulation, reuse and displacement and so on demand more and more, make double layer architecture more bloated trival, three-tier application architecture system arises at the historic moment. Three layer architecture architecture is inevitable outcome in the development of object-oriented thought. The three-tier architecture, is between client/server joined a "middle tier", also called component layer, together with the client and the server layer constitutes the three layers system [4]. From the development and application perspective, the three layer architecture than double or single structure has a greater advantage. Three layer structure is suitable for community development, each person can have different division of labor, to work together on doubling efficiency. Double or single application, every developer response system have deeper understanding, ability request is very high, three layer application, can be combined with various talents, only a few people on the system overall understanding, reduce the difficulty of development. Combining with the characteristics of curriculum management system design of three layer architecture is shown in Fig. 1.

(1) Presentation Layer: directly interact with the user, and responsible response to the user's request, generally refers to the system interface, used for data entry and data display, etc. The presentation layer of this system is mainly for "would secretary, would management, Course the teacher and Students in school, Other personnel" five types such as user provide operational services. Between the presentation layer and business layer through "Data exchange interface".

(2) Business Layer. It's between the presentation Layer and data Layer, responsible for handling user input information or to save the information sent to the data Layer, or Layer through data from the database read these data [5]. This layer can include some code for "business logic" to describe. The business layer is the bridge between the presentation layer and data layer, is responsible for 
data processing and transmission. This system provided by the business logic mainly includes "Data processing, Data insertion, Data update, Data delete, Data query, Data report", etc.

(3) Data Layer: to define and maintain Data's integrity and security, response to the request of logic Layer, is used to interact with the database. Perform data add, delete, modify and display and so on, all the data objects only cited in this layer. At the heart of the data access is a relational database system, also include other types of files, such as binary files, such as text documents or XML document. Between the business layer and Data layer through the Data access interface to interact.

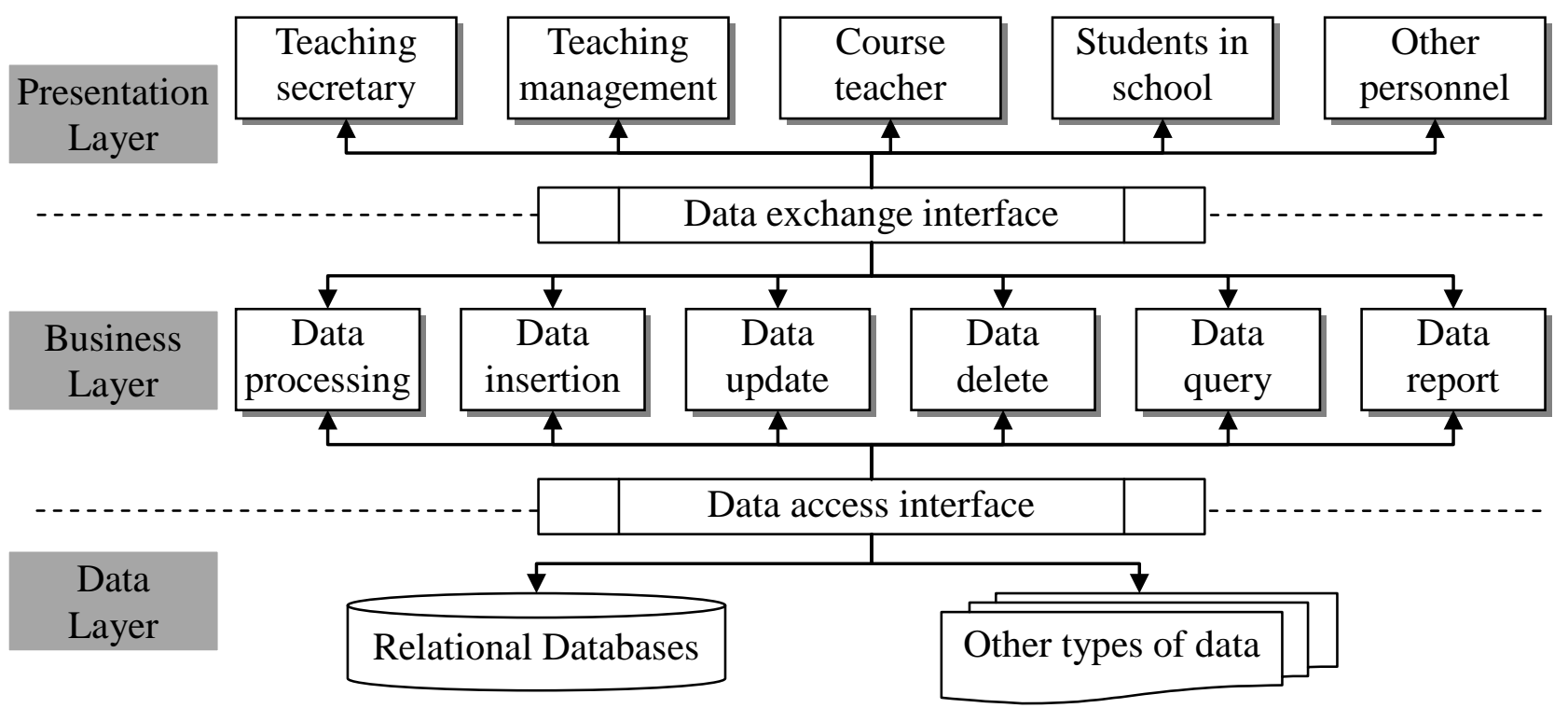

Fig. 1. Framework on curriculum management system

\section{System Functional Design}

System function design is functional decomposition process, from big to small, from coarse to fine, from top to bottom. Conceptually, upper function to control the lower, upper function more generally, the lower the specific function. Functional decomposition process is a from abstract to concrete, from complex to simple process. Function module can according to the specific situation of larger or smaller, decomposition to the minimum of each function module can be a program in the process, and the larger function module can complete a task is a set of procedures. With reference to previous research results [6,7], the system function design results are shown in Fig. 2.

(1) Web home page: the home page is the default user open a browser open Web pages, refers to a system starts to enter the first page, most Web applications as the file name is the homepage of the index, the default, the main or portal and extension. System is often home page editor is easy to understand the information provided by the system, and guide the user to browse other parts system.

(2) Curriculum management: is the core of the system to provide the function, main complete CRUD operations, namely the increase of processing data calculation, regain operations such as data, update, and delete data. Course information input, modify, and delete is the most important data operation, this system is closely related to the course of the teaching plan of inputting, modifying, and deleting operation is also more important data operation.

(3) System common: is for all applications for the System function. User registration must be conducted before using the system, you can download the system to provide the related resources, through the online help to understand the system to provide the functionality and usage, through personalized Settings sets the system to their familiar display or operating mode.

(4) Rights management: refers to according to the rules of the safety of the system Settings or security policy, users can access resources and can only access authorized. This system adopt the method of role permission management, role is a kind of collection of users, with the same 
operation permissions. Only to the role authorization, users belonging to the role, reduce the complexity of permission management.

(5) Basic information: a unified interface to the system maintenance, Basic information for convenient for information centralized management, reduce maintenance costs, enhancing the standardization of data. The basic information of this system maintenance including "Course nature information, Course code information, would the college information, Check mode information", etc.

(6) Query report: query data directly from the data source table or on the source table data only for simple calculations; Report data is the data source table by writing to the complex operation purpose, the purpose of data from table or for simple operation objective table data. This system provides the query statements including "Course information query, would plan query, Course information report, would plan report", etc.

\begin{tabular}{|c|c|}
\hline \multicolumn{2}{|c|}{ Curriculum management system } \\
\hline Web home page & Curriculum management \\
\hline Home page news & Course information input \\
\hline Important links & Curriculum information revision \\
\hline Function navigation & Course information delete \\
\hline Notification or notice & Teaching plan input \\
\hline Core toolbar & Teaching plan update \\
\hline System profile & Teaching plan delete \\
\hline System common & Rights management \\
\hline User registration & User login \\
\hline Resources download & Role management \\
\hline Online help & Certificate management \\
\hline Personalized settings & Role authorization \\
\hline Basic information & Query report \\
\hline Course nature information & Course information query \\
\hline Course code information & Teaching plan query \\
\hline Teaching college information & Course information report \\
\hline Check mode information & Teaching plan report \\
\hline
\end{tabular}

Fig. 2. Function on curriculum management system

\section{Conclusion}

Educational administration management system management system is to run modern methods of dealing with the teaching information system, through the network to realize educational administration collaboration and data sharing between departments, is the foundation of the modern educational administration of colleges and universities [8]. Curriculum management system is the core of the educational administration system function, able to manage the basic information of the whole school courses, arrange the class and class time and class classroom, management course examination results, and can realize the query statistics of the examination results. This article 
follows the principle and method of system engineering design, laid the foundation for the subsequent development of the system work.

\section{Acknowledgement}

This work is supported by 2013 annual social science planning fund project of Liaoning province (L13CTQ012): Research on investigation and cultivation for college students' reading tendency of digital resources; 2014 annual education science planning of Liaoning province (JG14DB019): Strategy research on teachers' teaching mode transformation and teaching ability in digital environment.

\section{References}

[1] R. Guo, L. P. Jiao, L. Cheng, "Analysis and Design of Course Management System Based on Web," Computer Knowledge and Technology, vol. 9, no. 15, pp. 3512-3513, 2013.

[2] D. Kang, "Design of course management system based on project teaching," Information systems engineering, vol. 27, no. 5, pp. 153-156, 2014.

[3] P. F. Li, "Design and implementation of management system for comprehensive course in university based on.NET," Master's degree of Yanshan University, 2012.

[4] Flighterstart's blog, "Introduction to the three tier architecture," http://blog.sina.com.cn/s/blog_ae7b72560101kvoq.html, 2016-4-11.

[5] Huijin's blog, "Three tier architecture: presentation layer business logic layer data access layer," http://blog.sina.com.cn/s/blog_4b02ab3b01011s4d.html, 2016-4-11.

[6] T. T. Hu, "Functional design of curriculum management system based on MVC model," Journal of Shanxi Coal-Mining Administrators College, vol. 26, no. 4, pp. 140-142, 2013.

[7] M. L. Cheng, "Discussion on Network Security of Course Management Information System Based on Internet," Computer Knowledge and Technology, vol. 12, no. 5, pp. 16-18, 2016.

[8] B. Y. Luan, "Design and implementation of Educational Administration Management System in University," Master's degree of Jilin University, 2013. 